October 29, 2018

CTP-MIT-3014

hep-th/yymmnn

\title{
On Marginal Deformations in Superstring Field Theory
}

\author{
Amer Iqbal ${ }^{\sharp}$, Asad Naqvi ${ }^{\dagger}$ \\ ${ }^{1}$ Center for Theoretical Physics, MIT \\ Cambridge, MA 02139, U.S.A.
}

\begin{abstract}
We use level truncated superstring field theory to obtain the effective potential for the Wilson line marginal deformation parameter which corresponds to the constant vacuum expectation value of the $U(1)$ gauge field on the Dbrane in a particular direction. We present results for both the BPS and the non-BPS D-brane. In the case of non-BPS D-brane the effective potential has branches corresponding to the extrema of the tachyon potential. In the branch with vanishing tachyon vev ( $M$-branch), the effective potential becomes flatter as the level of the approximation is increased. The branch which corresponds to the stable vacuum after the tachyon has condensed ( $V$-branch) exists only for a finite range of values of marginal deformation parameter. We use our results to find the mass of the gauge field in the stable tachyonic vacuum. We find this mass to be of a non-zero value which seems to stabilize as the level approximation is improved.
\end{abstract}

\footnotetext{
${ }^{1}$ Address from $1{ }^{\text {st }}$ September $2000:{ }^{\sharp}$ Department of Physics-Theory Group, The University of Texas at Austin, Austin, TX, 78712.

$\dagger$ Department of Physics and Astronomy, University of Pennsylvania, Philadelphia, Pennsylvannia, 19104
} 


\section{Contents}

1 Introduction 1

2 Marginal deformations and Level Approximation on a BPS D-brane 3

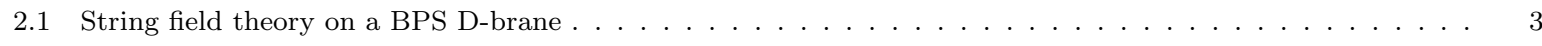

2.2 String fields for the Wilson line marginal deformation $\ldots \ldots \ldots \ldots$

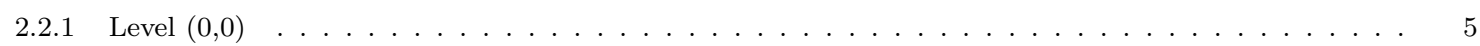

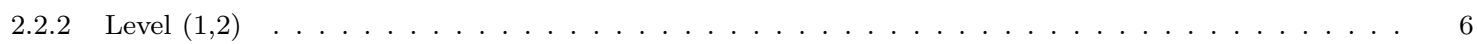

3 Marginal deformations and Level approximation on a non-BPS D-brane $\quad 8$

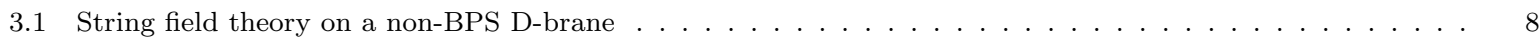

3.2 Marginal deformations and Level approximation $\ldots \ldots \ldots \ldots$

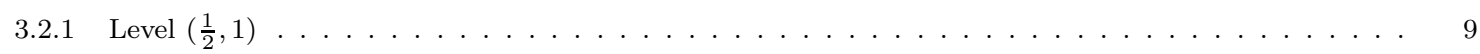

3.2 .2 Level $\left(\frac{1}{2}, 2\right) \ldots \ldots \ldots \ldots \ldots \ldots \ldots \ldots \ldots \ldots \ldots \ldots$

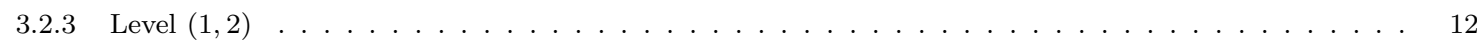

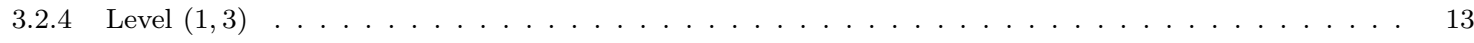

$3.2 .5 \quad$ Level $\left(\frac{3}{2}, 3\right) \ldots \ldots \ldots \ldots \ldots \ldots \ldots \ldots \ldots \ldots \ldots \ldots \ldots$

4 Mass of the $U(1)$ gauge field 17

5 Conclusions 18

\section{Introduction}

Recently, it has been realized that string field theory is a useful setup to address questions related to tachyon condensation in string theory [1]. Tachyonic systems, such as the open string tachyon on a D-brane in bosonic string theory as well as on a non-BPS D-brane or a D-brane anti D-brane pair in Type II string theory, have been analyzed in level truncated string field theory [2-9]. It has also been argued that a co-dimension one D-brane can be considered as the tachyonic lump in bosonic open string field theory [10-12]. Co-dimension two lump solutions in string field theory have also been explored in a recent papers $[13,14]$. Tachyon condensation in the D0/D4 system in the presence of NS B-field has also been studied, using the superstring field theory, in [15]. From the two dimensional conformal field theory point of view, vevs for the tachyonic field corresponds to adding relevant operators to the CFT [16]. In addition to the tachyon, D-branes have massless open string states some of which correspond to exactly marginal 
operators of the CFT. Such massless states, which correspond to marginal deformations, should correspond to flat directions in the potential of string field theory.

One familiar example of a marginal deformation of the CFT is the Wilson-line, a constant vev of a $U(1)$ gauge field along a compact direction. In string field theory, we expect a flat potential for the field $w$ corresponding to this marginal deformation. However, in the level truncation scheme, to any finite level, the potential is not expected to be exactly flat. In [17] Sen and Zwiebach calculated the potential, in the level truncated bosonic string field theory, for the marginal deformation parameter corresponding to the constant vev of the $U(1)$ gauge field. They determined the effective potential for $w$ by solving the equations of motion for all fields except $w$ in terms of $w$ and substituting in the potential. They showed that the effective potential becomes flatter as the higher level field and terms in the action are included. The effective potential, as expected, is not flat at any given level. However, as the approximation is improved, the potential becomes flatter.

In section 2, we calculate the effective potential for the Wilson line on a BPS D-brane in Type II string theory by using Berkovits' open superstring field theory $[18,19]$. We work in the level truncation scheme and calculate the potential first at the $(0,0)$ level and then at the $(1,2)$ level. By $(n, m)$ level approximation, we mean that we keep the states up to level $n$ and terms in the action up to level $m$. For the case of the BPS D-brane, we assign level to a state to be equal to its $L_{0}$ eigenvalue. This is different from the level assignments in section 3 as well as the assignments in $[4,6,7]$. At the level $(1,2)$, we did not find different branches of solutions in the BPS case.

In section 3, we analyze the case of the non-BPS D-brane. Here, the tachyon, in the $\mathrm{GSO}(-)$ sector survives and has $L_{0}$ eigenvalue $-\frac{1}{2}$. We assign level of a state with $L_{0}$ eigenvalue $h$ to be $h+\frac{1}{2}$ so that the tachyon has level 0. A major difference between the BPS and the non-BPS case is the existence of two distinct branches of solutions in the non-BPS case. One branch corresponds to zero vev of the tachyon field, $t,(M$-branch [17]) and the other branch corresponds to the true vacuum after the tachyon has condensed ( $V$-branch). That there is a $t=0$ branch for all values of $w$ is clear form the fact that GSO odd fields appear at least quadratically in the action hence a solution exists to the equations of motion with all $\mathrm{GSO}(-)$ fields set to zero. Hence the effective potential for $w$ in the $M$-branch is identical to that in the BPS case. However, in the non-BPS case, in addition to the solution with $t=0$, there are two solutions with non-zero vev for the tachyon but they differ from each other by a sign. This continues to be the case at non-zero values of $w$. This is different from the case of bosonic string field theory where there was no branch in which the tachyon vev was identically zero. The two branches in 
the bosonic case where not related to each other by an overall sign and were physically distinct. For the non-BPS D-brane, there are really three branches but two of them are trivially related to each other by $t \rightarrow-t$. As the value of $w$ increases, the $V$-branch merges with the $M$-branch at a particular value of $w$. For values of $w$ greater than this value, the $V$-branch does not exist. However, the $M$-branch continues to exist for all values of $w$ (at least in the level approximation up to level $(3 / 2,3)$ ). In section 4 we find the mass of the gauge field in the $V$-branch and as suggested by Taylor [20,21], we find a non-zero value which seems to stabilize as the level approximation is improved.

\section{Marginal deformations and Level Approximation on a BPS D-brane}

In this section, we will analyze the string fields and the string field action corresponding to CFT marginal deformations. In particular, we will single out a particular coordinate, $x_{9}$ along the world volume of a D-9 brane, and consider giving expectation value to the constant mode of the gauge field component $A_{9}$. This represents a marginal deformation of the BCFT describing the D-brane. We will find the string field corresponding to this deformation in the level approximation. For our purposes, whether or not the $x_{9}$ direction is compact is irrelevant; we give expectation values to the field independent in the $x_{9}$ direction, so that modes carrying non-zero momentum along the $x_{9}$ direction (and

other directions) are set to zero. We will use Berkovits' open superstring field theory for our analysis.

\subsection{String field theory on a BPS D-brane}

In $[22,18,3]$, a string field configuration in the $\mathrm{GSO}(+) \mathrm{NS}$ sector corresponds to a Grassmann even open string vertex operator $\Phi$ of ghost and picture number 0 [23] in the combined conformal field theory of a $c=15$ superconformal matter system, and the $b, c, \beta, \gamma$ ghost system with $c=-15$. Here, $b, c$ are the reparameterization ghosts and $\beta$, $\gamma$ are their superconformal partners. The $\beta, \gamma$ ghost system can be bosonized and can be replaced by ghost fields $\xi, \eta, \phi$, related to $\beta, \gamma$ through the relations

$$
\beta=\partial \xi e^{-\phi}, \quad \gamma=\eta e^{\phi} .
$$

The $\mathrm{SL}(2, \mathrm{R})$ invariant vacuum has zero ghost and picture number.

We shall denote by $\left\langle\prod_{i} A_{i}\right\rangle$ the correlation function of a set of vertex operators in the combined matter-ghost conformal field theory on the unit disk with open string vertex 
operators inserted on the boundary of the disk. These correlation functions are to be computed with the normalization

$$
\left\langle\xi(z) c \partial c \partial^{2} c(w) e^{-2 \phi(y)}\right\rangle=2
$$

The BRST operator is given by

$$
Q_{B}=\oint d z c\left(T_{m}+T_{\xi \eta}+T_{\phi}\right)+c \partial c b+\eta e^{\phi} G_{m}-\eta \partial \eta e^{2 \phi} b .
$$

Here $^{1}$

$$
T_{\xi \eta}=\partial \xi \eta, T_{\phi}=-\frac{1}{2} \partial \phi \partial \phi-\partial^{2} \phi, T_{m}=-\frac{1}{2} \partial X^{\mu} \partial X_{\mu}-\frac{1}{2} \psi^{\mu} \partial \psi_{\mu}, G_{m}=i \psi^{\mu} \partial X_{\mu}
$$

The normalization of $\psi^{\mu}, \partial X^{\mu}, \phi, \xi, \eta, b$ and $c$ are as follows:

$$
\begin{gathered}
\partial X^{\mu}(z) \partial X^{\nu}(w)=-\frac{\eta^{\mu \nu}}{(z-w)^{2}} \quad \psi^{\mu}(z) \psi^{\nu}(w)=\frac{\eta^{\mu \nu}}{z-w} \\
\xi(z) \eta(w) \simeq \frac{1}{z-w}, \quad b(z) c(w) \simeq \frac{1}{z-w}, \quad \partial \phi(z) \partial \phi(w) \simeq-\frac{1}{(z-w)^{2}} .
\end{gathered}
$$

We will denote by $\eta_{0}=\oint d z \eta(z)$ the zero mode of the field $\eta$ acting on the Hilbert space of matter ghost CFT.

The expansion of the string field action, relevant for our calculations is given by [4]

$$
\begin{aligned}
S=\frac{1}{g^{2}}\langle & \left\langle\frac{1}{2}\left(Q_{B} \Phi\right)\left(\eta_{0} \Phi\right)+\frac{1}{3}\left(Q_{B} \Phi\right) \Phi\left(\eta_{0} \Phi\right)+\frac{1}{12}\left(Q_{B} \Phi\right)\left(\Phi^{2}\left(\eta_{0} \Phi\right)-\Phi\left(\eta_{0} \Phi\right) \Phi\right)\right. \\
& +\frac{1}{60}\left(Q_{B} \Phi\right)\left(\Phi^{3}\left(\eta_{0} \Phi\right)-3 \Phi^{2}\left(\eta_{0} \Phi\right) \Phi\right) \\
& \left.\left.+\frac{1}{360}\left(Q_{B} \Phi\right)\left(\Phi^{4}\left(\eta_{0} \Phi\right)-4 \Phi^{3}\left(\eta_{0} \Phi\right) \Phi+3 \Phi^{2}\left(\eta_{0} \Phi\right) \Phi^{2}\right)\right\rangle\right\rangle .
\end{aligned}
$$

$\left\langle\left\langle A_{1}, \ldots A_{n}\right\rangle\right\rangle$ is defined as:

$$
\left\langle\left\langle A_{1} \ldots A_{n}\right\rangle\right\rangle=\left\langle f_{1}^{(n)} \circ A_{1}(0) \cdots f_{n}^{(n)} \circ A_{n}(0)\right\rangle .
$$

Here, $f \circ A$ for any function $f(z)$, denotes the conformal transform of $A$ by $f$, and

$$
f_{k}^{(n)}(z)=e^{\frac{2 \pi i(k-1)}{n}}\left(\frac{1+i z}{1-i z}\right)^{2 / n} \quad \text { for } \quad n \geq 1
$$

\footnotetext{
${ }^{1}$ We have set $\alpha^{\prime}=2$
} 


\subsection{String fields for the Wilson line marginal deformation}

To study the Wilson line marginal deformations in string field theory, we will restrict the string field to be in the subset of vertex operators of ghost and picture number zero, created from the matter stress tensor $\left(T_{m}(z)\right)$, its superconformal partner $\left(G_{m}(z)\right), \psi^{9}(z)$ and $\partial X^{9}(z)$ (where $x_{9}$ is the compact direction) and the ghost fields $b, c, \xi, \eta, \phi$. From now on, we will refer to $\psi^{9}$ and $\partial X^{9}$ as just $\psi$ and $\partial X$. In this section, the level of a string field component multiplying a vertex operator will be defined as the conformal weight $h$ of the vertex operator ${ }^{1}$. The string field action has a gauge invariance which can be used to choose a gauge in which

$$
b_{0} \Phi=0, \quad \xi_{0} \Phi=0,
$$

which is valid at least at the linearized level. All relevant states in the "small" Hilbert space can be obtained by acting with ghost number zero combinations of oscillators $\psi_{-r}$, $\alpha_{-n}, L_{n}, G_{-1-r}, b_{-n}, c_{1-n}, \beta_{-r}, \gamma_{-r}\left(n \geq 1\right.$ and $\left.r \geq \frac{1}{2}\right)$ on $|\Omega\rangle .|\Omega\rangle$ is the tachyon state (projected out by the GSO projection). The $b_{0} \Phi=0$ gauge condition allows us to ignore states with a $c_{0}$ oscillator in them (except when $L_{0}$ eigenvalue of the state is zero). As shown in [24] and used in [17], the string field theory action has a $Z_{2}$ symmetry which is a combination of twist symmetry and $X \rightarrow-X$ and $\psi \rightarrow-\psi$. Under twist symmetry, string field components associated with a vertex operator of dimension $h$ carry charge $(-1)^{h+1}$ for even $2 h$, and $(-1)^{h+\frac{1}{2}}$ for odd $2 h$. Thus it is possible to consistently restrict the string field $\Phi$ to be even under this $Z_{2}$ symmetry. We will now work in the level truncation scheme. $(n, m)$ approximation means that we keep states up to level $n$ and terms in the action up to level $m$. States up to level one which can contribute to the potential for the marginal deformation parameter are shown in Table 1. The boxed states are odd under the $\mathbf{Z}_{2}$ symmetry mentioned above and therefore can be ignored.

\subsubsection{Level $(0,0)$}

Since only $\mathrm{GSO}(+)$ sector survives on a BPS D-brane, at $L_{0}=0$, we can construct the state

$$
\psi_{-\frac{1}{2}}|\Omega\rangle
$$

Notice that the state $c_{0} \beta_{-\frac{1}{2}}|\Omega\rangle$ has $L_{0}$ eigenvalue zero. However, it is is odd under the $Z_{2}$ combination of twist and parity mentioned above so we can ignore it in our present calculation.

\footnotetext{
${ }^{1}$ This is different from the definition of level in the next section and also from the definition of level in $[4,6,7]$
} 


\begin{tabular}{|c|c|c|c|}
\hline$L_{0}$ & Level & Twist & State \\
\hline 0 & 0 & odd & $c_{0} \beta_{-\frac{1}{2}}|\widetilde{\Omega}\rangle, \psi_{-\frac{1}{2}}|\tilde{\Omega}\rangle$ \\
\hline 1 & 1 & even & $\begin{array}{c}\left.\left.\left|\psi_{-\frac{1}{2}} \beta_{-\frac{1}{2}} \gamma_{-\frac{1}{2}}\right| \widetilde{\Omega}\right\rangle\left|, \psi_{-\frac{3}{2}}\right| \widetilde{\Omega}\right\rangle, c_{-1} \beta_{-\frac{1}{2}}|\widetilde{\Omega}\rangle \\
b_{-1} \gamma_{-\frac{1}{2}}|\widetilde{\Omega}\rangle, G_{-\frac{3}{2}}|\widetilde{\Omega}\rangle, \alpha_{-1} \psi_{-\frac{1}{2}}|\widetilde{\Omega}\rangle\end{array}$ \\
\hline
\end{tabular}

Table 1: GSO even states of ghost number zero up to level one. Boxed states are odd under the $\mathbf{Z}_{2}$ symmetry mentioned before.

The state $\psi_{-\frac{1}{2}}|\Omega\rangle$ corresponds to the level 0 gauge field zero mode. The string field at this level is given by

$$
\Phi^{(0)}=w W
$$

where

$$
W=\xi \psi c e^{-\phi},
$$

is a primary field of weight zero. The string field action can be used to obtain the potential

$$
V(\Phi)=-S(\Phi)=-\left(2 \pi^{2} M\right) g^{2} S(\Phi) .
$$

Here $M=\frac{1}{2 \pi^{2} g^{2}}$ :

$$
V_{e f f}^{(0,0)}(w)=\left(2 \pi^{2} M\right) \frac{3}{8} w^{4}
$$

We note the absence of quadratic term for $w$. This is to be expected since $W$ is a marginal operator.

\subsubsection{Level $(1,2)$}

At $L_{0}=1$ (level 1 ), we get four new states ${ }^{2}$ :

$$
c_{-1} \beta_{-\frac{1}{2}}|\Omega\rangle, \quad b_{-1} \gamma_{-\frac{1}{2}}|\Omega\rangle, \quad G_{-\frac{3}{2}}|\Omega\rangle, \quad \psi_{-\frac{1}{2}} \alpha_{-1}|\Omega\rangle .
$$

The vertex operators corresponding to these states are:

$$
A=\xi \partial \xi c \partial^{2} c e^{-2 \phi}, E=\xi \eta, F=\xi G_{m} c e^{-\phi}, P=i \xi \psi \partial X c e^{-\phi},
$$

\footnotetext{
${ }^{2}$ We have not included states $\psi_{-\frac{1}{2}} \beta_{-\frac{1}{2}} \gamma_{-\frac{1}{2}}|\Omega\rangle$ and $\psi_{-\frac{3}{2}}|\Omega\rangle$ because they are odd under the combination of twist and parity symmetry.
} 
where $F$ and $P$ are primary fields of conformal weight 1 and $A$ and $E$ are not primary but transform as $(f(0)=u)[4]$ :

$$
\begin{aligned}
& f \circ A(0)=f^{\prime}(0)\left\{A(u)-\frac{f^{\prime \prime}(0)}{\left(f^{\prime}(0)\right)^{2}} c \partial c \xi \partial \xi e^{-2 \phi}(u)\right\} \\
& f \circ E(0)=f^{\prime}(0)\left\{E(u)-\frac{f^{\prime \prime}(0)}{2\left(f^{\prime}(0)\right)^{2}}\right\}
\end{aligned}
$$

The string field $\Phi$ is given by

$$
\Phi^{(1)}=w W+a A+f F+p P+e E .
$$

We can use this expansion in the string field action (2.4) and truncate the action to terms up to level 2 .

The result, with $S_{k}$ denoting the level $k$ terms in the action, is

$$
\begin{aligned}
g^{2} S_{0}= & -\frac{3}{8} w^{4} \\
g^{2} S_{1}= & \frac{4}{9} \sqrt{3}\left(w^{2} a-w^{2} f-w^{2} p\right)+\frac{1}{75} \sqrt{250+110 \sqrt{5}} w^{4} e \\
g^{2} S_{2}= & -\frac{1}{2} p^{2}-f p-2 a e-5 f^{2}-\frac{83}{24} w^{2} a e+\frac{95}{24} w^{2} e f-\frac{39}{8} w^{2} f^{2} \\
& -\frac{15}{16} w^{2} p^{2}+\frac{19}{48} w^{2} e p-\frac{15}{8} w^{2} f p+\frac{19}{48} w^{2} e^{2}-\frac{25}{177} w^{4} e^{2} . \\
& V(w, a, e, f, p)=-\left(2 \pi^{2} M\right) g^{2}\left(S_{0}+S_{1}+S_{2}\right) .
\end{aligned}
$$

The equations of motion, $\frac{\partial V}{\partial a}=\frac{\partial V}{\partial e}=\frac{\partial V}{\partial f}=\frac{\partial V}{\partial p}=0$ for $a, e, f, p$ can be solved to obtain $a, e, f, p$ as functions of $w$. These can be substituted in $V(w, a, e, f, p)$ to obtain the effective potential for $w$,

$$
V_{e f f}^{(1,2)}(w)=\left(2 \pi^{2} M\right) \frac{P(w)}{Q(w)}
$$

where

$$
\begin{aligned}
P(w)= & \frac{w^{4}}{106200}\left(29344814625 w^{8}-131631360 \sqrt{3} \sqrt{250+110 \sqrt{5}} w^{8}+68413522000 w^{6}\right. \\
& -296763392 \sqrt{250+110 \sqrt{5}} w^{6} \sqrt{3}-207831040 \sqrt{3} \sqrt{250+110 \sqrt{5}} w^{4} \\
& +55895956800 w^{4}+17269913600 w^{2}-46399488 \sqrt{3} \sqrt{250+110 \sqrt{5}} w^{2} \\
& +1232486400) \\
Q(w)= & 147456+915456 w^{2}+2085184 w^{4}+2049104 w^{6}+723345 w^{8}
\end{aligned}
$$


It is useful to expand $V_{e f f}^{(1,2)}(w)$ in powers of $w$. The result is

$$
\frac{V_{e f f}^{(1,2)}(w)}{M}=\frac{17}{216} w^{4}-\left(-\frac{199}{324}+\frac{2}{675} \sqrt{3} \sqrt{250+110 \sqrt{5}}\right) w^{6}+O\left(w^{8}\right)
$$

The coefficient of the $w^{4}$ term is $\frac{17}{216}$ in the $(1,2)$ approximation as compared to $\frac{3}{8}$ in the

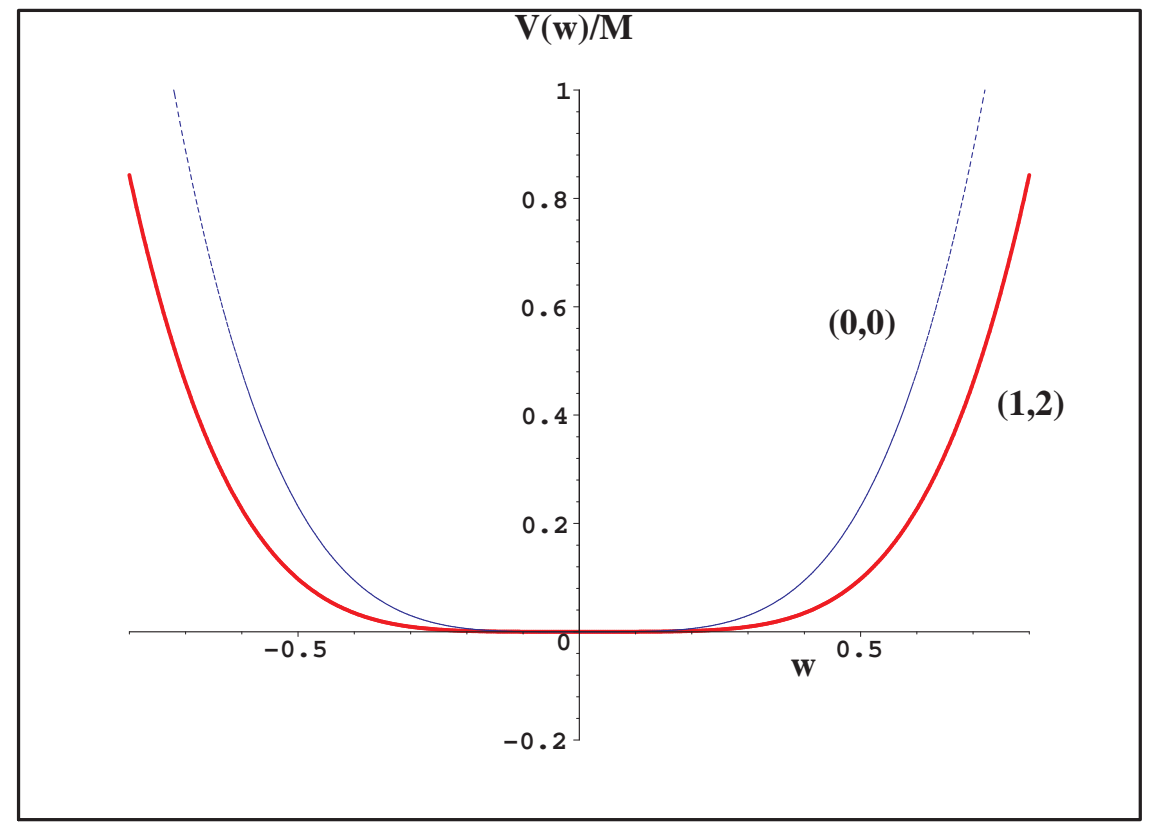

Figure 1: The effective potential for $w$ in the $(0,0)$ and $(1,2)$ approximations for a BPS D-brane.

$(0,0)$ approximation. The potential becomes flatter as we improve the approximation as shown in Fig 1. However, even at the $(1,2)$ level, we did not find multiple branches of solutions or any restriction on the value of $w$. This is different for the case of a D-brane in bosonic string theory discussed in [17].

\section{Marginal deformations and Level approximation on a non- BPS D-brane}

\subsection{String field theory on a non-BPS D-brane}

For a non-BPS D-brane, in addition to the $\mathrm{GSO}(+)$ sector, $\mathrm{GSO}(-)$ sector survives. The GSO $(-)$ states are Grassmann odd and to incorporate them in the algebraic structure of the previous section, internal $2 \times 2$ Chan Paton matrices were introduced in [4]. These 
are added both to the vertex operators, $Q_{B}$ and $\eta_{0}$ as discussed in detail in [4]. The complete string field, $Q_{B}$ and $\eta_{0}$ are thus written as

$$
\widehat{\Phi}=\Phi_{+} \otimes I+\Phi_{-} \otimes \sigma_{1}, \quad \widehat{Q}_{B}=Q_{B} \otimes \sigma_{3}, \quad \widehat{\eta}_{0}=\eta_{0} \otimes \sigma_{3} .
$$

where the subscripts on $\Phi$ denote the $(-1)^{F}$ eigenvalue of the vertex operator. The string field action for the non-BPS D-brane up to terms quintic in the string field is given by [3]:

$$
\begin{aligned}
S=\frac{1}{2 g^{2}} & \left\langle<\frac{1}{2}\left(\widehat{Q}_{B} \widehat{\Phi}\right)\left(\widehat{\eta}_{0} \widehat{\Phi}\right)+\frac{1}{3}\left(\widehat{Q}_{B} \widehat{\Phi}\right) \widehat{\Phi}\left(\widehat{\eta}_{0} \widehat{\Phi}\right)+\frac{1}{12}\left(\widehat{Q}_{B} \widehat{\Phi}\right)\left(\widehat{\Phi}^{2}\left(\widehat{\eta}_{0} \widehat{\Phi}\right)-\widehat{\Phi}\left(\widehat{\eta}_{0} \widehat{\Phi}\right) \widehat{\Phi}\right)\right. \\
& +\frac{1}{60}\left(\widehat{Q}_{B} \widehat{\Phi}\right)\left(\widehat{\Phi}^{3}\left(\widehat{\eta}_{0} \widehat{\Phi}\right)-3 \widehat{\Phi}^{2}\left(\widehat{\eta}_{0} \widehat{\Phi}\right) \widehat{\Phi}\right) \\
& \left.\left.+\frac{1}{360}\left(\widehat{Q}_{B} \widehat{\Phi}\right)\left(\widehat{\Phi}^{4}\left(\widehat{\eta}_{0} \widehat{\Phi}\right)-4 \widehat{\Phi}^{3}\left(\widehat{\eta}_{0} \widehat{\Phi}\right) \widehat{\Phi}+3 \widehat{\Phi}^{2}\left(\widehat{\eta}_{0} \widehat{\Phi}\right) \widehat{\Phi}^{2}\right)\right\rangle\right\rangle .
\end{aligned}
$$

where the correlation function appearing in the above action are defined as

$$
\left\langle\left\langle\widehat{A}_{1} \ldots \widehat{A}_{n}\right\rangle\right\rangle=\operatorname{Tr}\left\langle f_{1}^{(n)} \circ \widehat{A}_{1}(0) \cdots f_{n}^{(n)} \circ \widehat{A}_{n}(0)\right\rangle .
$$

\subsection{Marginal deformations and Level approximation}

In this section we will calculate the potential for the Wilson line corresponding to the constant gauge field in the $x^{9}$ direction. The corresponding massless state in the CFT is $\psi_{-\frac{1}{2}}|\widetilde{\Omega}\rangle$. We will denote the corresponding space-time field by $w$. As in the last section $\psi:=\psi^{9}$ and $\partial X:=\partial X^{9}$.

Since we are on a non-BPS D-brane we also have fields in the $\operatorname{GSO}(-)$ sector, especially the tachyon, which contribute to the potential for $w$. In the last section we defined the level of the fields to be equal to the $L_{0}$ eigenvalue of the corresponding CFT state. In this section we will have to modify the definition of the level due to the presence of the tachyon. A field corresponding to the CFT state of $L_{0}$ eigenvalue $h$ has level $h+\frac{1}{2}$.

In our calculation of the potential for the Wilson line we will restrict ourselves to fields of level less than or equal to $\frac{3}{2}$ and terms of level 3 or less in the action. The states and the vertex operators contributing to the potential are shown in the Table 2. Where in Table 2 the boxed states are not invariant $[17,24]$ under the combined $\mathbb{Z}_{2}$ twist transformation and $\left(\psi_{-n-\frac{1}{2}}, \alpha_{-n}\right) \mapsto\left(-\psi_{-n-\frac{1}{2}},-\alpha_{-n}\right)$. Thus, for example, the field corresponding to the state $c_{0} \beta_{-\frac{1}{2}}|\widetilde{\Omega}\rangle$ can be set to zero.

\subsubsection{Level $\left(\frac{1}{2}, 1\right)$}

The states contributing at this level and the corresponding vertex operators are

$$
|\widetilde{\Omega}\rangle, \quad \widehat{T}=\xi c e^{-\phi} \otimes \sigma_{1} ; \psi_{-\frac{1}{2}}|\widetilde{\Omega}\rangle, \widehat{W}=\xi \psi c e^{-\phi} \otimes I .
$$




\begin{tabular}{|c|c|c|c|c|}
\hline$L_{0}$ & Level & Twist & $G S O(+)$ & $G S O(-)$ \\
\hline$-\frac{1}{2}$ & 0 & even & & $|\tilde{\Omega}\rangle$ \\
\hline 0 & $\frac{1}{2}$ & odd & $c_{0} \beta_{-\frac{1}{2}}|\widetilde{\Omega}\rangle, \psi_{-\frac{1}{2}}|\tilde{\Omega}\rangle$ & \\
\hline$\frac{1}{2}$ & 1 & even & & $\alpha_{-1}|\tilde{\Omega}\rangle$ \\
\hline 1 & $\frac{3}{2}$ & odd & $\begin{array}{c}\psi_{-\frac{1}{2}} \beta_{-\frac{1}{2}} \gamma_{-\frac{1}{2}}|\widetilde{\Omega}\rangle, \psi_{-\frac{3}{2}}|\widetilde{\Omega}\rangle, c_{-1} \beta_{-\frac{1}{2}}|\widetilde{\Omega}\rangle \\
b_{-1} \gamma_{-\frac{1}{2}}|\widetilde{\Omega}\rangle, G_{-\frac{3}{2}}|\widetilde{\Omega}\rangle, \alpha_{-1} \psi_{-\frac{1}{2}}|\widetilde{\Omega}\rangle\end{array}$ & \\
\hline
\end{tabular}

Table 2: The states contributing to the potential for $w$ up to level $\frac{3}{2}$. The boxed states can be ignored since they are odd under the $\mathbf{Z}_{2}$ symmetry which is a combination of the twist and $\left(\alpha_{-n}, \psi_{-n-\frac{1}{2}}\right) \mapsto\left(-\alpha_{-n},-\psi_{-n-\frac{1}{2}}\right)$.

Plugging the string field $\widehat{\Phi}=t \widehat{T}+w \widehat{W}$ in the superstring action eq(3.2) and evaluating the terms up to level 1 we get

$$
g^{2} S=\frac{1}{4} t^{2}-\frac{1}{2} t^{4}+\left(\frac{1}{2}-\sqrt{2}\right) t^{2} w^{2} .
$$

Solving for the equation of motion of $t$ we get

$$
t=0, \text { and } t=t_{ \pm}= \pm \frac{1}{2} \sqrt{1-\frac{14}{1+2 \sqrt{2}} w^{2}}
$$

Thus we get

$$
\begin{aligned}
& V_{\text {eff }}^{\left(\frac{1}{2}, 1\right)}(w)=0 \text { for } t=0(M \text {-branch }), \\
& V_{\text {eff }}^{\left(\frac{1}{2}, 1\right)}(w)=-\frac{2 \pi^{2} M}{32}\left(1-\frac{14}{1+2 \sqrt{2}} w^{2}\right)^{2} \text { for } t=t_{ \pm}(V \text {-branch }) .
\end{aligned}
$$

These potentials are shown in the Fig 2 below. Note that in the tachyonic branch the allowed value of $w$ is restricted to the interval $[-0.52293,+0.52293]$. Also the value of the effective potential at $w=0$ in the tachyonic branch is equal to the value of tachyon potential at the minimum $(-0.617 M)$ calculated in [3]. 


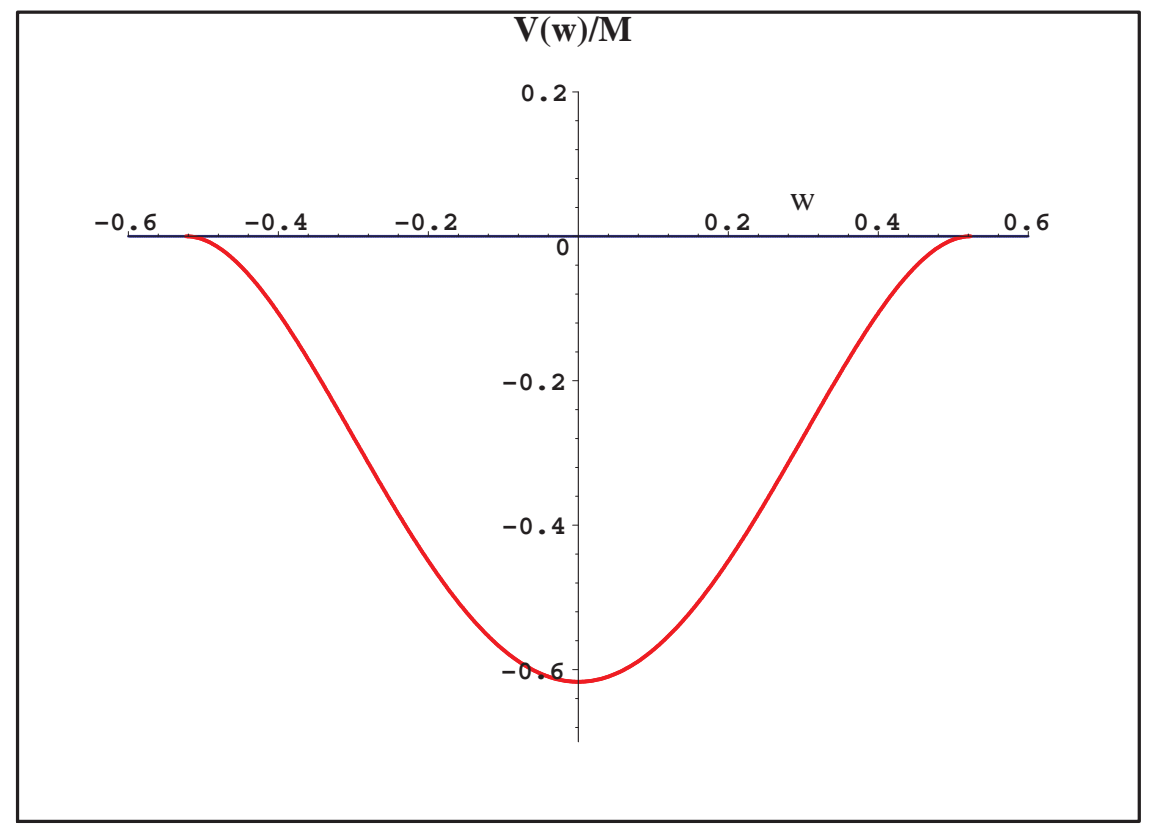

Figure 2: The effective potential for $w$ at level $\left(\frac{1}{2}, 1\right)$.

\subsubsection{Level $\left(\frac{1}{2}, 2\right)$}

In this approximation we keep the fields up to level $\frac{1}{2}$ and terms in the action up to level 2. This gives us one more term in the action,

$$
g^{2} S=\frac{1}{4} t^{2}-\frac{1}{2} t^{4}+\left(\frac{1}{2} \sqrt{2}\right) t^{2} w^{2}-\frac{3}{8} w^{4} .
$$

Solving for the equation of motion of $t$ we get,

$$
t=0, \quad t=t_{ \pm}= \pm \frac{1}{2} \sqrt{1-\frac{14}{1+2 \sqrt{2}} w^{2}}
$$

In the $M$-branch $(t=0)$ the effective potential for $w$ is

$$
V_{e f f}^{\left(\frac{1}{2}, 2\right)}(w)=\left(2 \pi^{2} M\right) \frac{3}{8} w^{4}, w \in[-\infty,+\infty](M \text {-branch }) .
$$

In the $V$-branch in which $t$ is non-zero we get

$$
V_{e f f}^{\left(\frac{1}{2}, 2\right)}(w)=\frac{\pi^{2} M}{16(1+2 \sqrt{2})^{2}}\left\{(48 \sqrt{2}-88) w^{4}+(56 \sqrt{2}+28) w^{2}-4 \sqrt{2}-9\right)(V \text {-branch }) .
$$

and the range of $w$ is the same as it was for the $V$-branch in the $\left(\frac{1}{2}, 1\right)$ approximation. Fig 3 shows the effective potentials in the $M$ and the $V$-branch. 


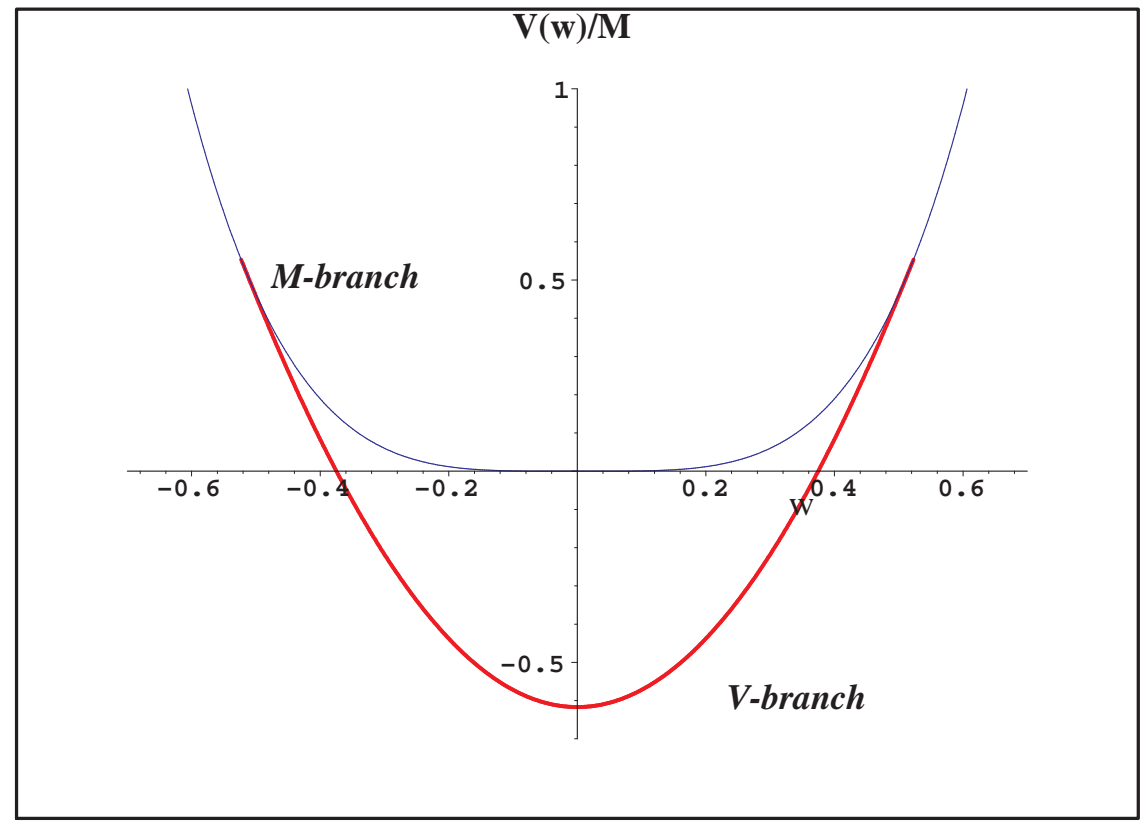

Figure 3: The effective potential for $w$ at level $\left(\frac{1}{2}, 2\right)$.

\subsubsection{Level $(1,2)$}

At this level we have on more state contributing to the potential for $w$,

$$
\alpha_{-1}|\widetilde{\Omega}\rangle, \quad \widehat{Y}=i \xi \partial X c e^{-\phi} \otimes \sigma_{1} .
$$

The string field is now given by $\widehat{\Phi}=t \widehat{T}+w \widehat{W}+y \widehat{Y}$. Keeping terms up to level 2 in the action we get,

$$
g^{2} S=\frac{1}{4} t^{2}-\frac{1}{2} t^{4}+\left(\frac{1}{2}-\sqrt{2}\right) t^{2} w^{2}-\frac{3}{8} w^{4}-k y t-\frac{1}{4} y^{2}-\frac{5}{4} t^{2} y^{2} .
$$

We solve for $y$ and $t$ as a function of $w$ by solving for the equations of motion $\frac{\partial S}{\partial y}=\frac{\partial S}{\partial t}=0$ and get the effective potential for $w$. The effective potential in the two branches is shown in Fig 4 below.

The effective potential in the $t=0$ branch is the same as the $\left(\frac{1}{2}, 2\right)$ case,

$$
V_{e f f}^{(1,2)}(w)=\left(2 \pi^{2} M\right) \frac{3}{8} w^{4} \quad(M \text {-branch }) .
$$

Although we found an exact analytic expression for the effective potential in the $V$-branch, we will not list it here as it is quite complicated. We will just give the first few terms in its taylor expansion:

$V_{e f f}^{(1,2)}(w)=M\left(-0.61685+2.31822 w^{2}+2.33284 w^{4}+4.450471 w^{6}+O\left(w^{8}\right)\right) \quad(V$-branch $)$. 


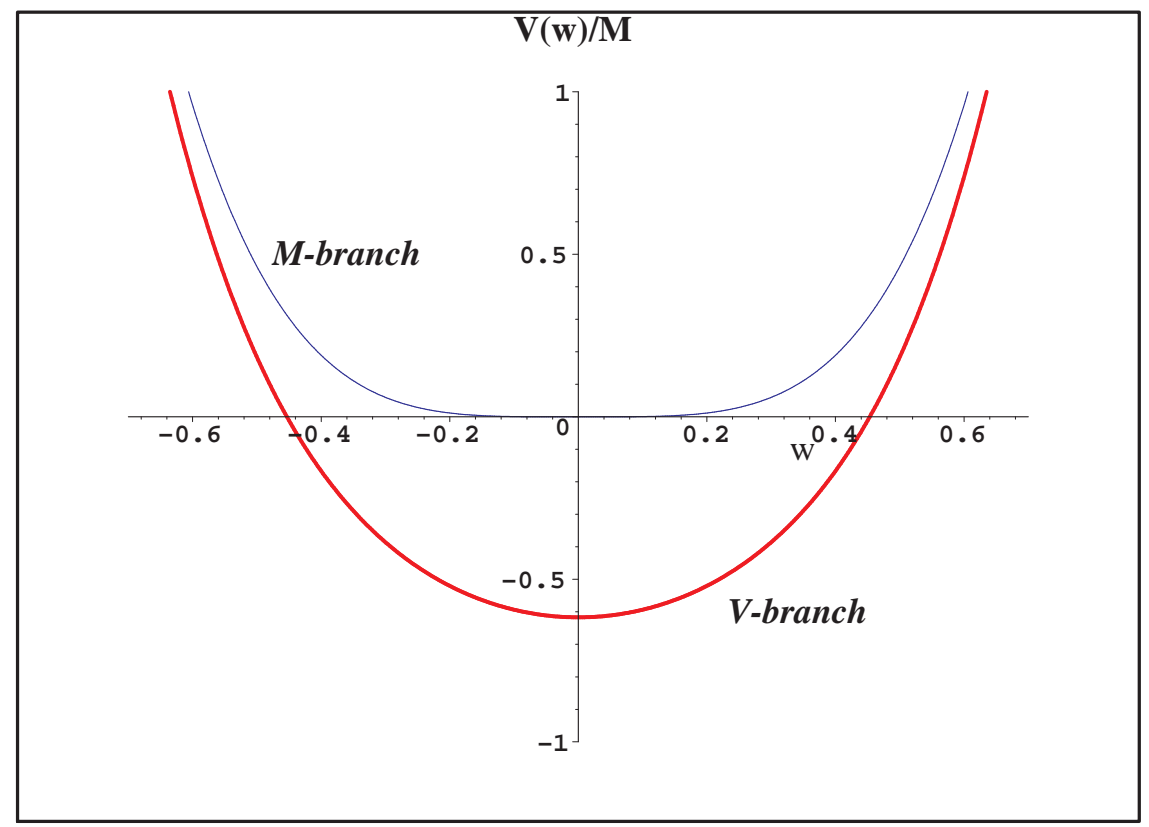

Figure 4: The effective potential for $w$ at level $(1,2)$.

\subsubsection{Level $(1,3)$}

Including level three terms in the action we obtain one more term as compared to the action at level $(1,2)$,

$$
\begin{aligned}
g^{2} S & =\frac{1}{4} t^{2}-\frac{1}{2} t^{4}+\left(\frac{1}{2}-\sqrt{2}\right) t^{2} w^{2}-\frac{3}{8} w^{4}-w y t-\frac{1}{4} y^{2} \\
& -\frac{5}{4} t^{2} y^{2}+\left(\frac{1}{8}-\frac{1}{2} \sqrt{2}\right) w^{2} y^{2}
\end{aligned}
$$

As before, we can eliminate $y$ and $t$ by using their equations of motion to obtain the effective potential for $w$. In the $M$-branch $t=y=0$ and the effective potential for $w$ is $\left(2 \pi^{2} M\right) \frac{3}{8} w^{4}$. In the $V$-branch $t$ and $y$ are non-zero. In this branch the value of $w$ is restricted to lie in the range [-0.7306, 0.7306]. At ends of this interval, the $V$-branch touches the $M$-branch. The expression for the effective potential in the $V$-branch is fairly long so we will just give the first few terms in its taylor expansion:

$V_{e f f}^{(1,3)}=M\left(-0.61685+2.31822 w^{2}+4.60253 w^{4}+2.82462 w^{6}+O\left(w^{8}\right)\right) \quad(V$-branch $)$.

The graph of the effective potential in the two branches is shown in figure 5 . 


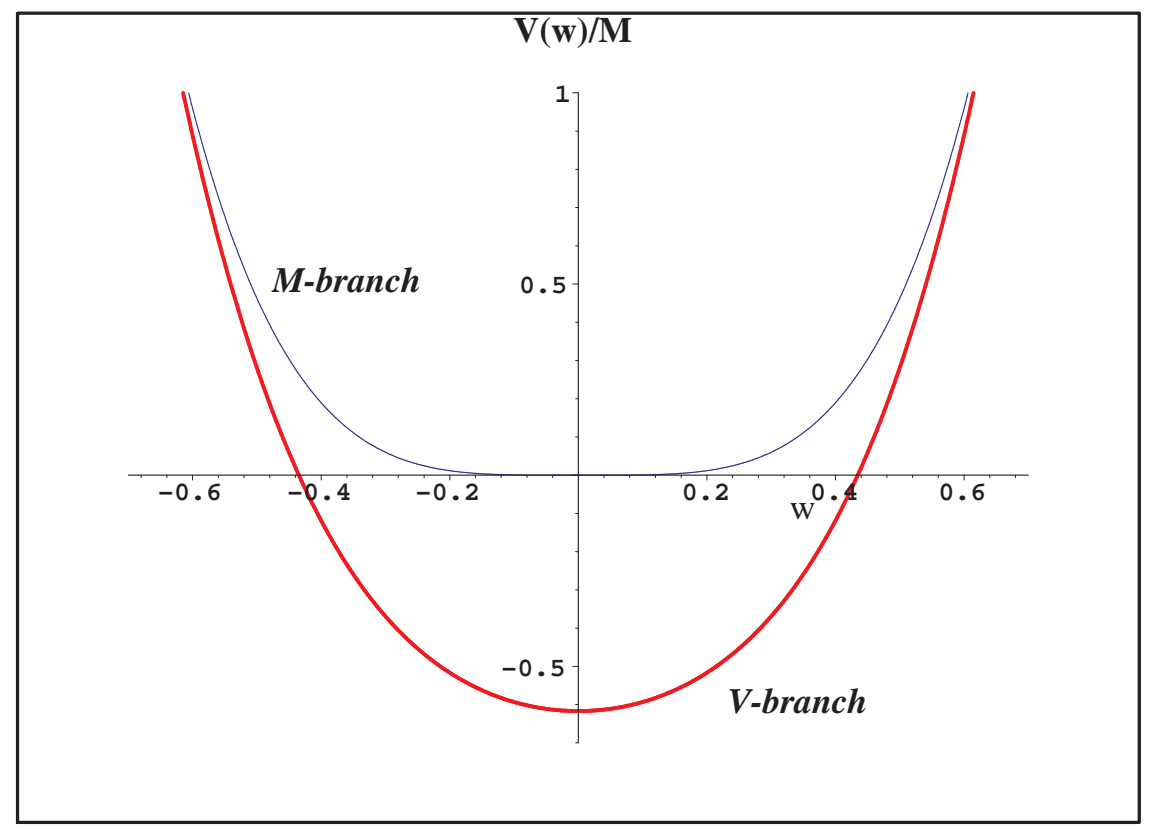

Figure 5: The effective potential for $w$ at level $(1,3)$.

\subsubsection{Level $\left(\frac{3}{2}, 3\right)$}

At this level we have four new fields contributing to the potential for $w$,

$$
\left\{c_{-1} \beta_{-\frac{1}{2}}, b_{-1} \gamma_{-\frac{1}{2}}, G_{-\frac{3}{2}}, \alpha_{-1} \psi_{-\frac{1}{2}}\right\}|\widetilde{\Omega}\rangle
$$

with vertex operators

$$
\widehat{A}=\xi \partial \xi c \partial^{2} c e^{-2 \phi} \otimes I, \widehat{E}=\xi \eta \otimes I, \widehat{F}=\xi G_{m} c e^{-\phi} \otimes I, \widehat{P}=i \xi \psi \partial X c e^{-\phi} \otimes I .
$$

The string field up to this level is given by

$$
\widehat{\Phi}=t \widehat{T}+w \widehat{W}+y \widehat{Y}+a \widehat{A}+e \widehat{E}+f \widehat{F}+p \widehat{P} \text {. }
$$

Using this string field and keeping only terms up to level 3 in the action we get,

$$
\begin{aligned}
g^{2} S_{0} & =\frac{1}{4} t^{2}-\frac{1}{2} t^{4} \\
g^{2} S_{\frac{1}{2}} & =0 \\
g^{2} S_{1} & =\left(\frac{1}{2}-\sqrt{2}\right) t^{2} w^{2} \\
g^{2} S_{\frac{3}{2}} & =-w y t+a t^{2}+\frac{1}{4} e t^{2}+\frac{5}{96} \sqrt{50+22 \sqrt{5}} e t^{4} \\
g^{2} S_{2} & =-\frac{3}{8} w^{4}-\frac{1}{4} y^{2}-\frac{5}{4} t^{2} y^{2}
\end{aligned}
$$




$$
\begin{aligned}
& g^{2} S_{\frac{5}{2}}=\frac{1}{24}(9+5 \sqrt{5}) e w^{2} t^{2}+\frac{4 \sqrt{3}}{9}\left(a w^{2}-f w^{2}-p w^{2}\right) \\
& g^{2} S_{3}=-2 a e-p f-\frac{5}{18} e^{2} t^{4}-\frac{1}{12}(3+40 \sqrt{2}) a e t^{2}+\frac{5}{12}(10 \sqrt{2}-1) e f t^{2} \\
&+\frac{1}{24}(10 \sqrt{2}-1) e p t^{2}-\frac{1}{4}(4 \sqrt{2}-1) f p t^{2}-5 f^{2}-\frac{1}{2} p^{2}-\frac{1}{8}(4 \sqrt{2}-1) p^{2} t^{2} \\
&+\left(\frac{1}{\sqrt{2}}-\frac{1}{24}\right) e^{2} t^{2}+\left(\frac{1}{8}-\frac{1}{2} \sqrt{2}\right) y^{2} w^{2}-\frac{5}{4}(4 \sqrt{2}-1) f^{2} t^{2}+\frac{5}{12}(\sqrt{2}-2) e y w t \\
&+\left(\frac{3}{4}-\frac{9}{8} \sqrt{2}\right)(f y w t+p y w t) . \\
& V(w, t, y, a, e, f, p)=-\left(2 \pi^{2} M\right) g^{2} S
\end{aligned}
$$

We solve equations of motions for $y, a, e, f, p$ to obtain the vevs of these fields as functions of $w$ and $t$. Substituting these vevs in $V(w, t, y, a, e, f, p)$, we obtain an effective potential $V_{\text {eff }}(w, t)$ for $w$ and $t$. This is shown in Fig 6 . To obtain the effective potential for $w$, we have to solve the equation $\frac{\partial V(w, t)}{\partial t}=0$ for $t$ as a function of $w$. There is alway a solution $t=0$ to this equation. In this branch, the effective potential is given by

$$
V_{e f f}^{\left(\frac{3}{2}, 3\right)}(w)=\frac{17}{216} w^{4} \quad M \text {-branch. }
$$

In the $V$-branch $t$ is non-zero and we numerically find the set of points (other than $t=0$ ) $(w, t)$ such that $\frac{\partial V(w, t)}{\partial t}=0$. These points are shown in the Fig 7 . 


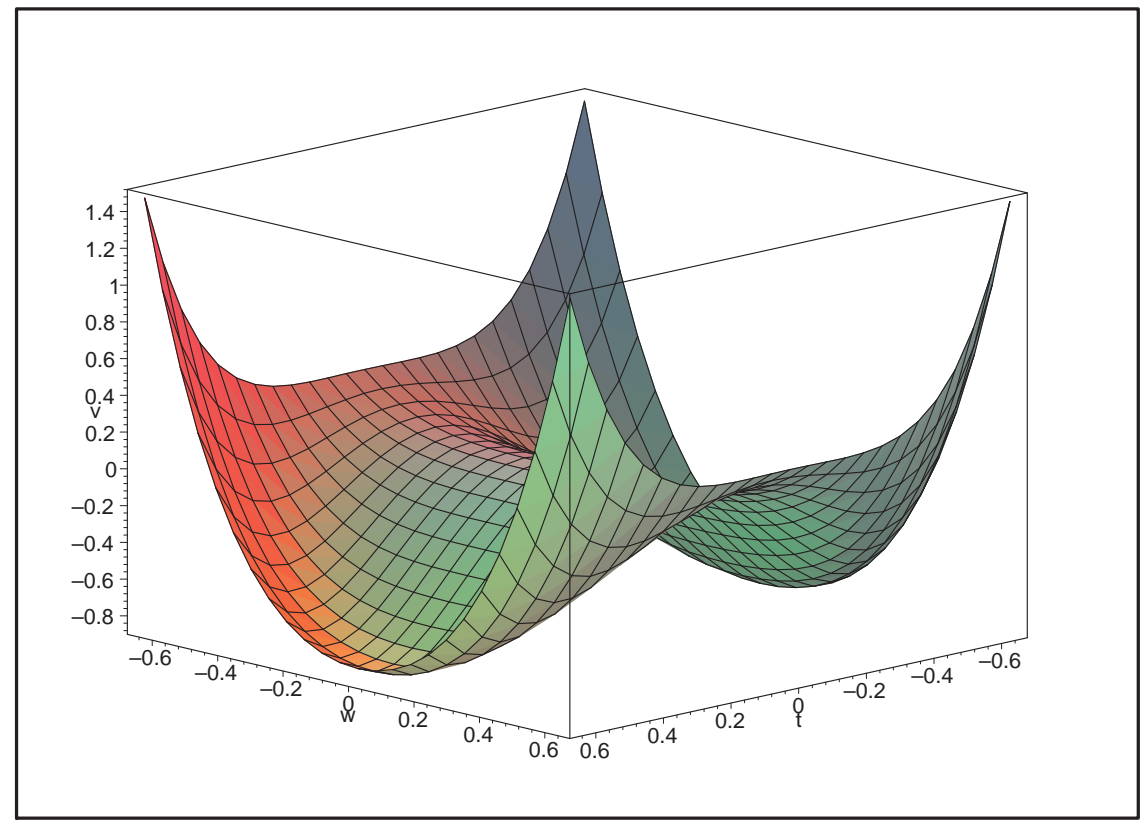

Figure 6: $\frac{V(w, t)}{M}$ at level $\left(\frac{3}{2}, 3\right)$.

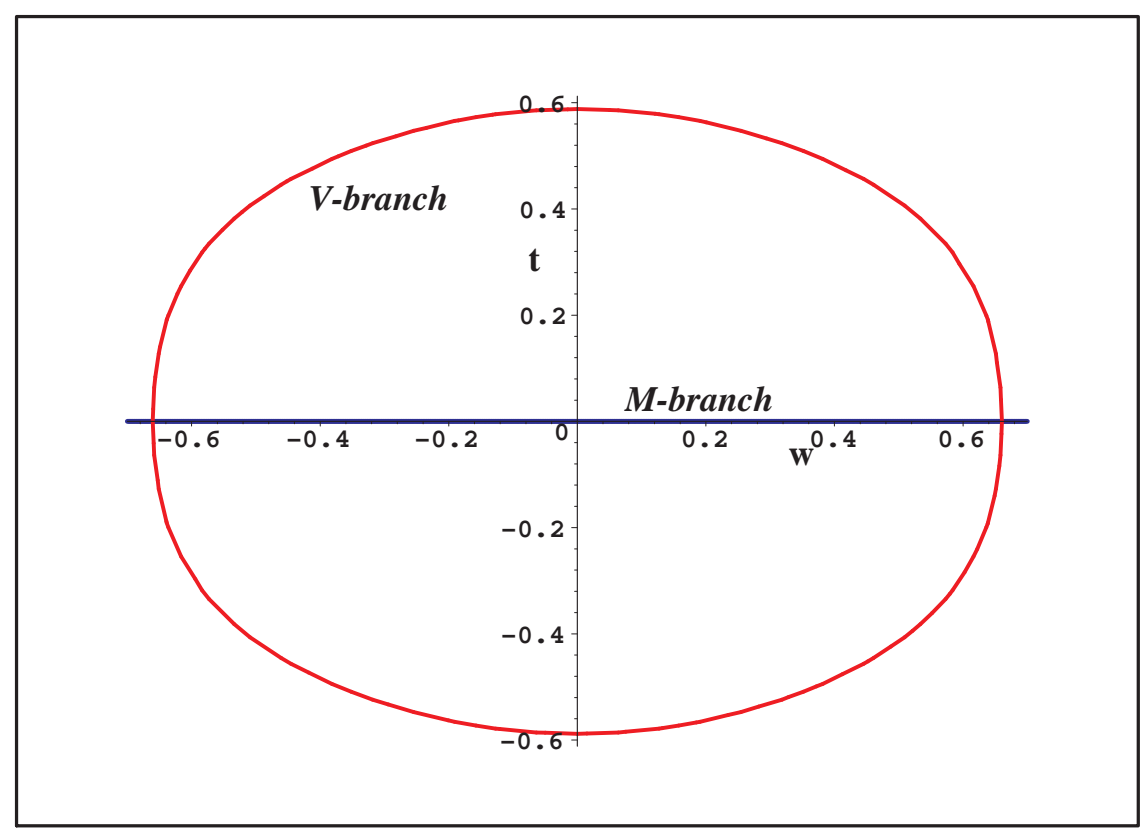

Figure 7: The set of points $(w, t)$ such that $\frac{\partial V(w, t)}{\partial t}=0$. The value of $w$ is restricted to lie in the range $\approx[-0.6617,0.6617]$ and at the end points of the interval the $V$-branch touches the $M$-branch. 
From Fig 7 we see that in the $V$-branch, the value of $w$ is restricted to lie in the range $[-0.6617,0.6617]$. We numerically find the effective potential for $w$ in the $V$-branch at this level. It is shown in Fig 8 below. Note that the value of the effective potential at $w=0$ is -0.85446 which is equal to the value of the tachyon potential at its minimum at this level [4].

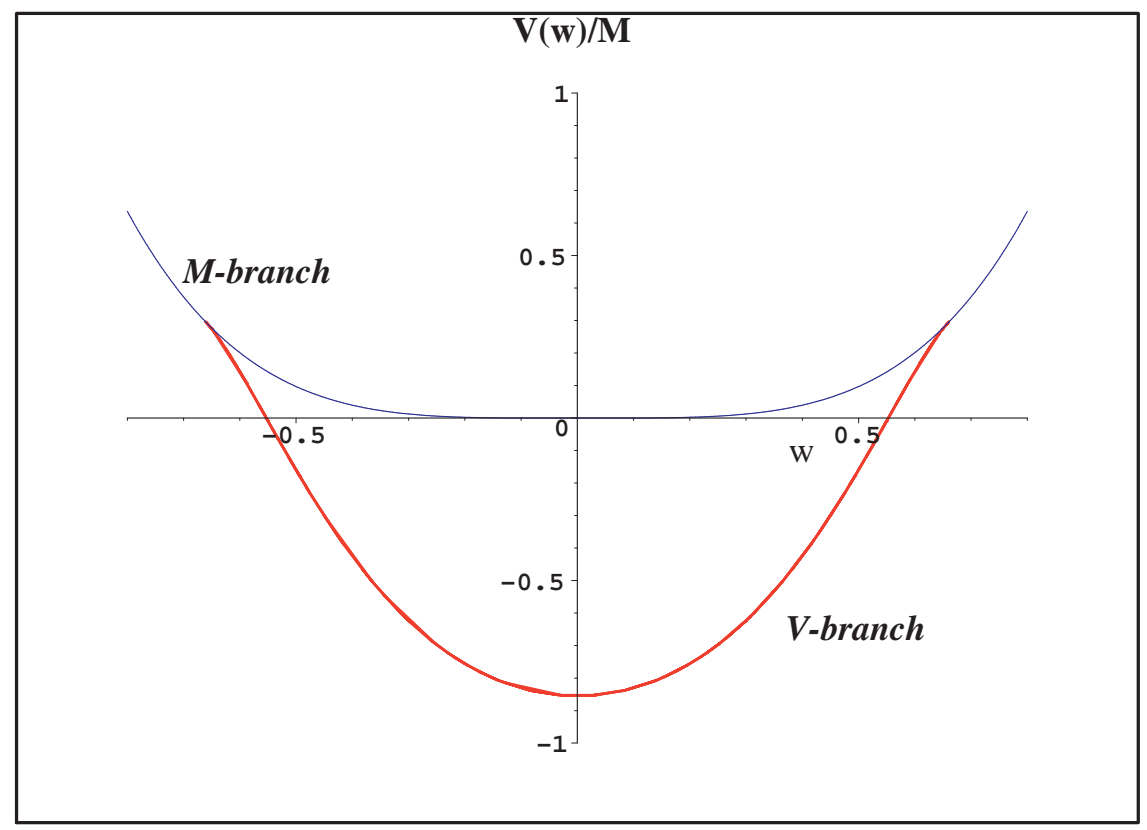

Figure 8: The effective potential for $w$ at level $\left(\frac{3}{2}, 3\right)$.

\section{Mass of the $U(1)$ gauge field}

The puzzle involving the fate of the $U(1)$ gauge field living on the non-BPS D-brane, as the D-brane annihilates, has been discussed by various authors[1, 25, 26, 20]. In [20] Taylor calculated the terms quadratic in the gauge field using level truncation in the bosonic string field theory. He showed that the eigenvalues of the mass matrix approach a non-zero value as higher and higher levels are included. Using the effective potential in the $V$-branch determined in the last section we calculate the mass of the gauge field in the stable tachyonic vacuum. The mass (squared) is the coefficient of the quaratic term in the expansion of the effective action in powers of $w$. The result is given in Table 3 . 


\begin{tabular}{||c|c|c||}
\hline Level & $V(0)$ & $m^{2}=\frac{1}{2} V^{\prime \prime}(0)$ \\
\hline \hline$\left(\frac{1}{2}, 1\right)$ & -0.61685 & 4.51146 \\
\hline$\left(\frac{1}{2}, 2\right)$ & -0.61685 & 4.51146 \\
\hline$(1,2)$ & -0.61685 & 2.31822 \\
\hline$(1,3)$ & -0.61685 & 2.31822 \\
\hline$\left(\frac{3}{2}, 3\right)$ & -0.85446 & 2.38223 \\
\hline
\end{tabular}

Table 3: The mass of the gauge field in different level approximations. The values in the second column are equal to the values, at that level, of the tachyon potential at its minimum.

It seems that the mass of the gauge field approaches a non-zero stable value as the level approximation is improved. However, we will have to include higher level fields and terms in the action to get more evidence for this fact.

\section{Conclusions}

In this paper we calculated the effective potential for the marginal deformation parameter corresponding to the constant vev of the $U(1)$ gauge field in the level truncated superstring field theory. We saw that for the case of BPS D-brane that there are no multiple branches of the effective potential at level $(1,2)$. Therefore their was no restriction on the range of the marginal deformation parameter $w$. This was clear from the beginning since terms at this level were at most quadratic in the fields $a, e, f$ and $p$ so that equations of motion are linear in these variables and there is a unique vev of these fields in terms of $w$. It is possible that if we include level three terms in the action there might be multiple branches of the effective potential since, in this case, we will have terms cubic in the fields $a, e, f$ and $p$ in the action. For example terms like $f^{3}, p^{3}$ and $f^{2} p$ are non-zero and solving for their equation of motion may give multiple solutions. Although determining the contribution of these cubic terms to the action is relatively easy, solving for their equations of motion to obtain the effective potential for $w$ seems difficult. However, to find out if the range of $w$ is restricted in the BPS case and if there are multiple branches of solutions coming from massive string fields, such a calculation should be performed.

For the non-BPS D-brane, the existence of two branches of solutions is due to to the fact that the tachyon potential at this level has two extrema (up to signs). In the 
unstable vacuum corresponding to zero tachyon vev ( $M$-branch) the effective potential for $w$ is not restricted and in the approximation we considered is purely quartic with the coefficient becoming smaller as the higher level contributions are included. In the $M$-branch, all GSO(-) fields are set to zero and the effective potential is identical to the BPS case (the effective potential in the BPS case in section 2 is more complicated than the potential found in the $M$-branch of the non-BPS case in section 3 ; this is because the level assignments used in the two cases are different). The $V$-branch of the effective potential corresponds to the non-zero tachyon vev and its range is restricted. At the end point of range the $V$-branch merges into the $M$-branch. At $w=0$, the value of the potential in the $V$-branch is the same as the value of tachyon potential at the minimum calculated in $[3,4]$.

Using the effective potential in the $V$-branch we also calculate the mass of the gauge field in the stable tachyonic vacuum. Our results seem to suggest that the gauge field acquires a non-zero mass after the tachyon condenses. However, to obtain better evidence of this, the level approximation should be pushed further.

\section{Acknowledgements}

We thank Barton Zwiebach for useful discussions. This research was supported in part by the US Department of Energy under contract \#DE-FC02-94ER40818.

\section{References}

[1] A. Sen, "Universality of Tachyon Potential", JHEP 9912, 027 (1999), [hepth/9911116].

[2] A. Sen, B. Zwiebach, "Tachyon condensation in string field theory", JHEP 0003 (2000) 002, [hep-th/9912249].

[3] N. Berkovits, "The Tachyon Potential in Open Neveu-Schwarz String Field Theory", JHEP 0004 (2000) 022, [hep-th/0001084].

[4] N. Berkovits, A. Sen, B. Zwiebach, "Tachyon Condensation in Superstring Field Theory", [hep-th/0002211].

[5] N. Moeller, W. Taylor, "Level Truncation and the tachyon in open bosonic string field theory", Nucl. Phys. B583 (2000) 105-144, [hep-th/0002237]. 
[6] P. De Smet, J. Raeymaekers, "Level Four Approximation to the Tachyon Potential in Superstring Field Theory", JHEP 0005 (2000) 051, [hep-th/0003220].

[7] A. Iqbal, A. Naqvi, "Tachyon Condensation on a non-BPS D-brane", [hepth/0004015].

[8] P. De Smet, J. Raeymaekers, "The Tachyon Potential in Witten's Superstring Field Theory", [hep-th/0004112].

[9] L. Rastelli, B. Zwiebach, "Tachyon potentials, star products and universality", [hepth/0006240].

[10] J.A. harvey, P. Kraus, "D-Branes as Unstable Lumps in Bosonic Open String Field Theory", JHEP 0004 (2000) 012, [hep-th/0002117].

[11] R. de Mello Koch, A. Jevicki, M. Mihailescu, R.Tatar, "Lumps and P-branes in Open String Field Theory", [hep-th/0003031].

[12] N. Moeller, A. Sen, B. Zwiebach, "D-branes as tachyon Lumps in String Field Theory", [hep-th/0005036].

[13] R. De Mello Koch, J.P. Rodrigues, "Lumps in level truncated open string field theory", [hep-th/0008053].

[14] N. Moeller, "Codimension two lump solutions in string field theory and tachyonic theories", [hep-th/0008101].

[15] J. David, "Tachyon condensation in D0/D4 system", [hep-th/0007235].

[16] J.A. Harvey, D. Kutasov, E. J. Martinec, "On the relevance of tachyons", [hepth/0003101].

[17] A. Sen, B. Zwiebach, "Large Marginal Deformations in String Field Theory", [hepth/0007153].

[18] N. Berkovits, "A New Approach to Superstring Field Theory", Fortsch. Phys. 48 (2000) 31-36, [hep-th/9912121].

[19] N. Berkovits, "Super-Poincare Covariant Quantization of the Superstring", JHEP 0004 (2000) 018, [hep-th/0001035].

[20] W. Taylor, "Mass generation from tachyon condensation for vector fields on Dbranes', [hep-th/0008033]. 
[21] W. Taylor, "D-brane effective field theory from string field theory", [hep-th/0001201].

[22] N. Berkovits, "Super-Poincare Invariant Superstring Field Theory," Nucl. Phys. B450 (1995) 90, [hep-th/9503099].

[23] D. Friedan, E. Martinec, and S. Shenker, "Conformal Invariance, Supersymmetry, and String Theory," Nucl. Phys. B271 (1986) 93.

[24] M. Gaberdiel, B. Zwiebach, "Tensor Constructions of Open String Theories I: Foundations", Nucl. Phys. B505 (1997) 569-624, [hep-th/9705038].

[25] P.Yi, "Membranes from five-branes and fundamental strings from Dp-branes", Nucl. Phys. B550 (1999) 214, [hep-th/9901159].

[26] O. Bergman, K. Hori, P. Yi, "Confinement on the brane", Nucl. Phys. B580 (2000)289, [hep-th/0002223]. 